\title{
Sarma Phase in Trapped Unbalanced Fermi Gases
}

\author{
K. B. Gubbels, ${ }^{*}$ M. W. J. Romans, and H. T. C. Stoof \\ Institute for Theoretical Physics, Utrecht University, Leuvenlaan 4, 3584 CE Utrecht, The Netherlands
}

(Received 13 June 2006; published 22 November 2006)

\begin{abstract}
We consider a trapped unbalanced Fermi gas at nonzero temperatures where the superfluid Sarma phase is stable. We determine, in particular, the phase boundaries between the superfluid, normal, and phaseseparated regions of the trapped unbalanced Fermi mixture. We show that the physics of the Sarma phase is sufficient to understand the recent observations of Zwierlein et al. [Science 311, 492 (2006); Nature (London) 442, 54 (2006)] and indicate how the apparent contradictions between this experiment and the experiment of Partridge et al. [Science 311, 503 (2006)] may be resolved.
\end{abstract}

DOI: $10.1103 /$ PhysRevLett.97.210402

Introduction. - In the past two years, impressive experimental progress has been made in the field of ultracold Fermi gases, in part due to the possibility to tune the interatomic interaction strength by means of a Feshbach resonance. This has led to the study of the crossover between the Bose-Einstein condensation of diatomic molecules and the Bose-Einstein condensation of atomic Cooper pairs, the so-called BEC-BCS crossover [1-3]. Most recently, two experimental groups have gone even a step further by obtaining also full control over the polarization of the Fermi mixture. This has allowed for the study of degenerate Fermi gases with imbalanced spin populations, which is a topic of great interest in many areas of physics ranging from condensed-matter physics to nuclear and astroparticle physics. These pioneering experiments by Zwierlein et al. [4,5] and Partridge et al. [6] induced a flurry of theoretical activity [7-21].

The ${ }^{6} \mathrm{Li}$ experiments of Zwierlein et al. and Partridge et al. both revealed that superfluidity in an ultracold Fermi gas is maintained upon going to an unequal mixture of two spin states. However, rather contradictory results are obtained by both experimental groups for the behavior of the Fermi mixture as a function of the population imbalance. Zwierlein et al. observe a phase transition between the superfluid phase and the normal phase at a high critical polarization of about $70 \%$, whereas Partridge et al. seem to observe a transition between two different superfluid phases at a low critical polarization of about $10 \%$. One important aim of the present Letter is, therefore, to propose a single theoretical picture in which the qualitative differences in the observations by the two experimental groups can be understood as two different sides of the same coin. Based on this picture, we also make a detailed quantitative comparison with the experiments of Zwierlein et al.

The main results of our mean-field calculations are presented in Fig. 1. Here we show the universal phase diagram of a trapped Fermi gas in the unitarity limit as a function of temperature and polarization. This phase diagram is universal in the sense that it does not depend on the total number of fermions or the trap geometry. Note that in determining the phase diagram we have neglected fluctua-
PACS numbers: 05.30.Fk, 03.75.- $\mathrm{b}, 39.25 .+\mathrm{k}, 67.40 .-\mathrm{w}$

tions, which are known to be quantitatively important in the unitarity limit. However, fluctuations are not expected to alter the topology of the phase diagram in this case. Figure 1 reveals that there is a tricritical point in the trapped Fermi mixture, which is well known for the homogeneous case $[20,22,23]$ but has, to the best of our knowledge, not been studied yet for the harmonically trapped situation. In the normal phase, the gas is in its normal state throughout the trap. In the Sarma phase, the Fermi gas has a shell structure, in which the core of the trapped gas is superfluid, whereas the outer region is normal. Furthermore, the normal-to-superfluid transition as a function of the position in the trap is of second order. Since the superfluid order parameter $\Delta$ vanishes continuously at the transition, we have for nonzero polarizations always a region in the trap where $|\Delta|$ is so small that it results in a gapless superfluid with negative quasiparticle excitation

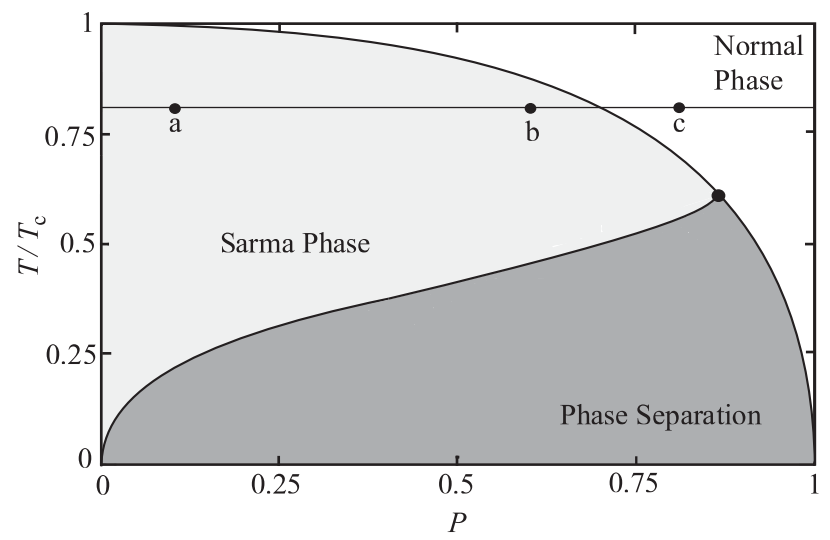

FIG. 1. Universal phase diagram of a trapped unbalanced Fermi gas in the unitarity limit. The polarization $P$ is given by $\left(N_{+}-N_{-}\right) /\left(N_{+}+N_{-}\right)$, where $N_{ \pm}$designates the number of fermions in each hyperfine state of the Fermi mixture. The temperature $T$ is scaled with the critical temperature $T_{c}$ of the balanced Fermi gas. The solid horizontal line gives the temperature that is used for the comparison with the experiments of Zwierlein et al. presented in Figs. 2 and 3. The points a, b, and c correspond to the polarizations used in the density profiles of Figs. 2(a)-2(c). 
energies, as first studied by Sarma [22]. Since $|\Delta|$ increases towards the center of the trap, it is also possible for small polarizations and low temperatures that the superfluid becomes gapped in the center of the trap. This leads to a gapped BCS superfluid core with a gapless Sarma superfluid and normal shell surrounding it [24]. Finally, in the phase-separated region of the phase diagram, the superfluid core and the normal shell of the gas are separated by a firstorder transition as a function of position, which implies that $\Delta$ goes discontinuously to zero at a certain equipotential surface in the trap.

Figure 1 allows for a natural explanation of the qualitative differences in the observations by the two experimental groups. More precisely, we will argue in the second part of this Letter that the experiments of Zwierlein et al. have observed the transition from the normal phase to the Sarma phase, implying that these experiments have been performed above the temperature of the tricritical point. Moreover, we suggest that the experiments of Partridge et al. have been performed in the temperature regime below the tricritical point, since these experiments appear to see the transition between a non-phase-separated and a phase-separated superfluid phase. Note that this explanation is fundamentally different from the proposal of Machida et al., who obtain a different phase diagram by considering the Fulde-Ferrell-Larkin-Ovchinnikov (FFLO) phase, which has a spatially varying superfluid order parameter, rather than phase separation [16]. As a result, they obtain a Lifshitz point instead of a tricritical point. They argue that both experimental groups operate beneath this Lifshitz point, making it difficult to explain the qualitative differences observed by both groups. Moreover, there is presently no experimental indication for the presence of the FFLO phase. Finally, we stress that the local-density approximation that was used to obtain the phase diagram in Fig. 1 is not sufficiently accurate to describe all aspects of the experiments of Partridge et al. [12,13,15]. For this reason, we consider here from now on only the experiment of Zwierlein et al., where a quantitative comparison with our theory turns out to be possible.

Universal phase diagram. - To obtain the phase diagram in Fig. 1, we use the mean-field theory for the Sarma phase in the local-density approximation as described by Houbiers et al. [25]. There the mean-field theory was applied to superfluid ${ }^{6} \mathrm{Li}$ in the BCS limit. Here we incorporated the relevant physics of the unitarity limit by using a generalization of the approach put forward by Fregoso and Baym [26]. As a result, we end up with the following thermodynamic potential:

$$
\begin{aligned}
\Omega= & \sum_{\mathbf{k}}\left(\epsilon_{\mathbf{k}}-\mu^{\prime}-\hbar \omega_{\mathbf{k}}+\frac{|\Delta|^{2}}{2 \epsilon_{\mathbf{k}}}\right) \\
& -\frac{1}{\beta} \sum_{\mathbf{k}, \sigma} \ln \left(1+e^{-\beta \hbar \omega_{\mathbf{k}, \sigma}}\right)-\frac{1}{2} \sum_{\sigma} N_{\sigma} \hbar \Sigma_{\sigma},
\end{aligned}
$$

where the atomic dispersion is $\epsilon_{\mathbf{k}}=\hbar^{2} \mathbf{k}^{2} / 2 m$, with $m$ the fermionic mass, $\Delta$ is the BCS order parameter, $\sigma= \pm$ denotes the two hyperfine states of the Fermi mixture, $N_{\sigma}$ is the number of atoms in each hyperfine state, and $\beta=1 / k_{B} T$. Moreover, the average renormalized chemical potential $\mu^{\prime}$ is given by $\mu^{\prime}=\left(\mu_{-}^{\prime}+\mu_{+}^{\prime}\right) / 2$, and the dispersions $\hbar \omega_{\mathbf{k}, \sigma}$ of the Bogoliubov quasiparticles satisfy $\hbar \omega_{\mathbf{k}, \sigma}=\sigma\left(\mu_{-}^{\prime}-\mu_{+}^{\prime}\right) / 2+\hbar \omega_{\mathbf{k}}$, with $\hbar \omega_{\mathbf{k}}=$ $\sqrt{\left(\epsilon_{\mathbf{k}}-\mu^{\prime}\right)^{2}+|\bar{\Delta}|^{2}}$. Finally, the densities $n_{\sigma}=N_{\sigma} / V$ are given by

$$
n_{\sigma}=\frac{1}{V} \sum_{\mathbf{k}}\left\{\left|u_{\mathbf{k}}\right|^{2} N\left(\hbar \omega_{\mathbf{k}, \sigma}\right)+\left|v_{\mathbf{k}}\right|^{2}\left[1-N\left(\hbar \omega_{\mathbf{k},-\sigma}\right)\right]\right\},
$$

where $V$ is the volume of the mixture and $N\left(\hbar \omega_{\mathbf{k}, \sigma}\right)=$ $1 /\left[\exp \left(\beta \hbar \omega_{\mathbf{k}, \sigma}\right)+1\right]$ are the Fermi distributions for the Bogoliubov quasiparticles. The BCS coherence factors $\left|u_{\mathbf{k}}\right|^{2}$ and $\left|v_{\mathbf{k}}\right|^{2}$ are determined by the relations $\left|u_{\mathbf{k}}\right|^{2}=$ $\left[1+\left(\epsilon_{\mathbf{k}}-\mu^{\prime}\right) / \hbar \omega_{\mathbf{k}}\right] / 2$ and $\left|u_{\mathbf{k}}\right|^{2}+\left|v_{\mathbf{k}}\right|^{2}=1$.

The renormalized chemical potentials are given by $\mu_{\sigma}^{\prime}=\mu_{\sigma}-\hbar \Sigma_{\sigma}$, where $\mu_{\sigma}$ are the chemical potentials and the self-energies are approximated at unitarity by

$$
\hbar \Sigma_{\sigma}=\frac{3 \pi^{2} \hbar^{2}}{m}\left(\beta-\beta_{\mathrm{BCS}}\right) \sqrt{\frac{\hbar^{2}}{m} \frac{1+\beta_{\mathrm{BCS}}}{2 \mu^{\prime}}} n_{-\sigma} .
$$

This expression can be understood as follows. Since the average kinetic energy of the atoms involved in the interaction is given by $2 \mu^{\prime}$, the effective interaction strength between the atoms is expected to be proportional to $1 / \sqrt{2 \mu^{\prime}}$ [26]. To understand also the proportionality constant, we make use of the fact that, in the case of equal density and at zero temperature, Eq. (2) results in $\mu_{\sigma}^{\prime}=$ $\left(1+\beta_{\mathrm{BCS}}\right) \epsilon_{F}$, where we introduced the Fermi energy $\epsilon_{F}$ of a balanced Fermi gas, and we also recall that in the BCS theory $\beta_{\mathrm{BCS}} \simeq-0.48$ [13]. Substituting this result for $\mu_{\sigma}^{\prime}$ into Eq. (3), we obtain for the chemical potential $\mu_{\sigma}=$ $\mu_{\sigma}^{\prime}+\hbar \Sigma_{\sigma}=(1+\beta) \epsilon_{F}$, which agrees with the exact result from Monte Carlo calculations for $\beta \simeq-0.59$ [27,28].

Taking the derivative of the thermodynamic potential with respect to $\Delta^{*}$ and equating it to zero gives us the BCS gap equation

$$
\frac{1}{V} \sum_{\mathbf{k}}\left[\frac{1-N\left(\hbar \omega_{\mathbf{k},+}\right)-N\left(\hbar \omega_{\mathbf{k},-}\right)}{2 \hbar \omega_{\mathbf{k}}}-\frac{1}{2 \epsilon_{\mathbf{k}}}\right]=0 .
$$

Note that in deriving this gap equation we did not differentiate the last term in the right-hand side of Eq. (1). Differentiating also this term results in fluctuation corrections to the mean-field theory, on which we comment at the end of this Letter.

The above theory is valid for a homogenous Fermi mixture in the unitarity limit. To account for the presence of an axially symmetric trapping potential $V(\mathbf{x})=$ $m\left(\omega_{\perp} r^{2}+\omega_{z} z^{2}\right) / 2$, we use the local-density approxima- 
tion. This means that the theory is locally homogeneous with a spatially varying chemical potential. As a result, the local renormalized chemical potential is given by $\mu_{\sigma}^{\prime}(\mathbf{x})=$ $\mu_{\sigma}-V(\mathbf{x})-\hbar \Sigma_{\sigma}(\mathbf{x})$. For a balanced Fermi gas at zero temperature, we thus have $\mu_{\sigma}-V(\mathbf{x})=(1+\beta) \epsilon_{F}(\mathbf{x})$, and therefore we retrieve, as desired, the exact density profile in this case. Note that in the outer region of the gas cloud the renormalized chemical potentials become negative, so that $2 \mu^{\prime}(\mathbf{x})$ in Eq. (3) is no longer a good measure for the average kinetic energy of the interacting fermions. Therefore, we then take $3 k_{B} T$ instead of $2 \mu^{\prime}$ as an appropriate measure for the kinetic energy.

We are now in the position to explain how we obtain the phase diagram in Fig. 1. We first determine the line between the normal and the two superfluid phases. This is achieved by solving the BCS gap equation in the center of the trap and finding the temperature at which the BCS order parameter vanishes. Inspection of the thermodynamic potential reveals that the vanishing of the order parameter can occur continuously or discontinuously, i.e., by a second-order or a first-order phase transition. If the transition is of first (second) order, we go from the normal to the phase-separated (Sarma) phase. At the tricritical point, these two different kinds of transitions merge.

So far, we looked only at the center of the trap, but the tricritical condition can also be satisfied at a certain equipotential surface outside the center of the trap. This gives us a point on the Sarma-to-phase-separation line. To see this, consider a point on this line and raise the temperature slightly. This changes the tricritical transition outside the center of the trap into a second-order transition slightly closer to the center of the trap, which means that the gas is in the Sarma phase. In a similar way, a slightly lower temperature leads to a first-order transition as a function of position in the trap, i.e., to the phase-separated phase.

Comparison with experiment. -We now compare our theory with the experiments of Zwierlein et al. First, we show in Fig. 2 three typical density profiles of the gas: two in the superfluid Sarma phase and one in the normal phase.
Using different methods, similar density profiles have recently also been obtained by Yi and Duan [11] and Chien et al. [19]. The most striking feature in the density profiles is the "bulge" in the minority and majority profiles in the Sarma phase. This bulge is a direct consequence of the presence of the condensate of Cooper pairs and was indeed one of the most important findings of the experiment. It shows that for an unbalanced unitarity gas, in contrast to an unbalanced Fermi gas in the BCS limit [25], the condensate of Cooper pairs has a very strong effect on the atomic density profiles. In the normal phase, the attractive effects of the self-energies given in Eq. (3) also slightly increase the central densities of the two species. However, this occurs always in a smooth featureless manner, whereas in the experiments of Zwierlein et al. a distinct feature is seen in the majority density profile at the edge of the minority cloud. This may be due to the fact that our mean-field-like self-energies are intended to exactly incorporate the $\beta$ parameter of the balanced unitarity gas but only approximately account for the strong correlations in the normal phase of the unbalanced unitarity gas. Another explanation could be that in the experiment the density profiles are determined after an expansion. This affects, in particular, the majority density profile, since the outside of the majority cloud expands ballistically, whereas the inner part inside the minority cloud expands hydrodynamically and thus faster than the outer part. The expansion is therefore not determined by a single scale factor, and this may lead to a pileup of atoms in the transition region.

Besides the above qualitative comparison, we can also make a quantitative comparison with the experiment of Zwierlein et al. by determining the radial size of the Cooper-pair condensate and the radial sizes of the minority and majority gas clouds. The radial size of the Cooper-pair condensate follows directly from the point where $\Delta(\mathbf{x})=$ 0 , but the determination of the minority and majority radii is somewhat more complicated, because we are working at a nonzero temperature and the density profiles always have a Gaussian tail. For simplicity, we determine these radial
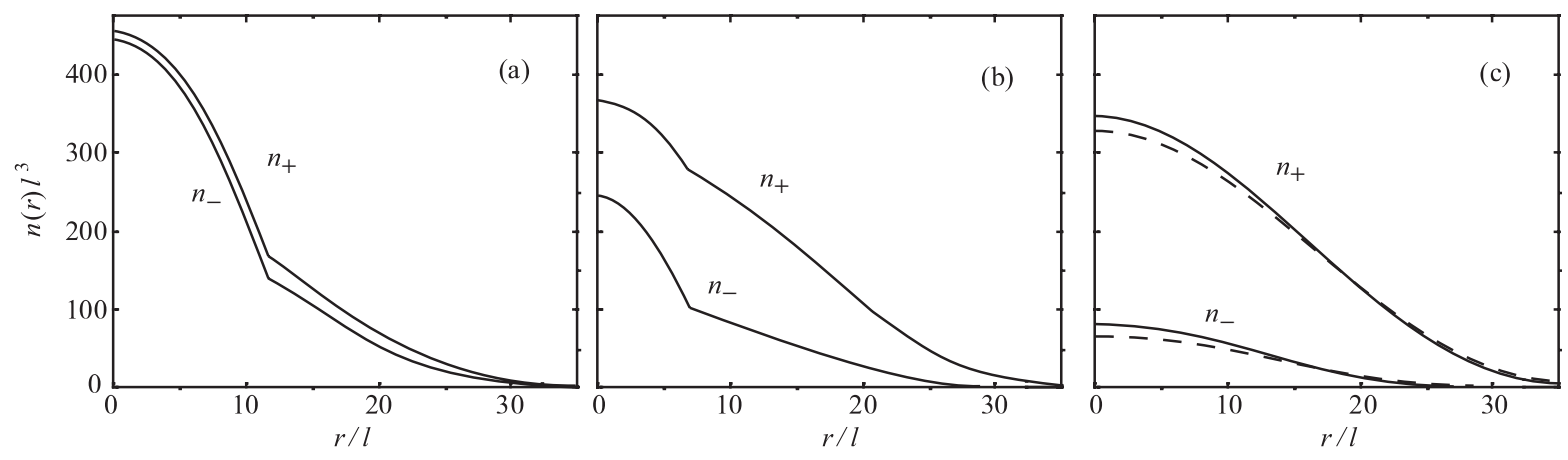

FIG. 2. Typical density profiles for trapped unbalanced Fermi gas above the tricritical point. The axial and radial directions of the trap are scaled in such a manner that the trap becomes effectively isotropic with a frequency $\omega=\left(\omega_{\perp}^{2} \omega_{z}\right)^{1 / 3}$ and a size $l=(\hbar / m \omega)^{1 / 2}$. The temperature and the polarizations used for the three panels are indicated in Fig. 1 . The total number of atoms is $N=1.4 \times 10^{7}$. The dashed lines in (c) show the ideal gas results. 


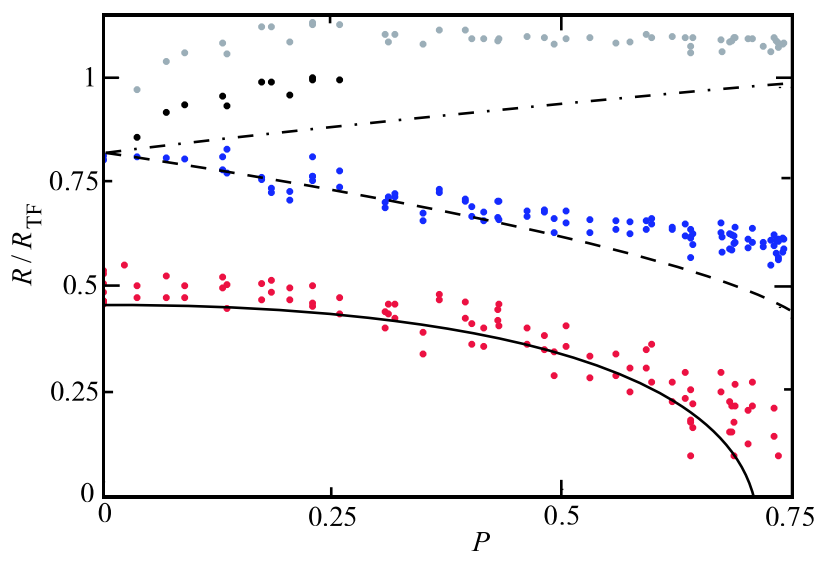

FIG. 3 (color online). The radial size $R$ of the Cooper-pair condensate (solid line), and the minority (dashed line) and majority (dotted-dashed line) gas clouds as a function of polarization for the temperature shown in Fig. 1. All radial sizes are scaled with the radial Thomas-Fermi radius of the balanced Fermi gas $R_{\mathrm{TF}}$. Also shown are the experimental data of Zwierlein et al. [5]. For the radial size of the majority cloud, the black points assume a hydrodynamic expansion, whereas the gray points assume a ballistic expansion.

sizes by the conditions $\mu_{\sigma}^{\prime}(\mathbf{x})=0$, which give the correct results at $T=0$ but underestimate the radial size of the density profiles at nonzero temperatures. The results of this procedure together with the experimental data are shown in Fig. 3. In general, the agreement with experiment is very good, which confirms our picture that the experiment is operating above the tricritical point. As expected, at small polarizations the radial size of the majority cloud agrees best with the experimental data obtained by assuming a hydrodynamic expansion, whereas for large polarizations our results approach the experimental data obtained by assuming a ballistic expansion.

Discussion. - The main remaining problem of our theory is the high absolute value of the temperatures above the tricritical point. The calculated temperatures are typically a factor of 5 higher than what is found in the experiments. An important consequence of the high temperature is that at the Sarma-to-normal transition the densities of the two spin states are not equal in the center of the trap, in contrast to what is found in experiments. The high absolute value of the temperature is the result of neglecting fluctuations that substantially shift down the tricritical temperature. Including fluctuations in the case of a balanced Fermi gas reduces the critical temperature in the unitarity limit by a factor of 3 , and fluctuations are expected to be even more important in the unbalanced case. One reason for the latter is that the Sarma phase is a gapless superfluid, whereas the BCS phase at $P=0$ has a gap. However, theoretically, the study of fluctuation effects is rather challenging for an unbalanced Fermi mixture at unitarity, since the usual Nozières-Schmitt-Rink approach [3] has some unphysical features in this case [20]. Therefore, we are developing a more advanced theory including fluctuations. This will determine the location of the tricritical point more accurately but, as mentioned previously, is not expected to alter the topology of the phase diagram.

We thank Randy Hulet and Martin Zwierlein for stimulating discussions. This work is supported by the Stichting voor Fundamenteel Onderzoek der Materie (FOM) and the Nederlandse Organisatie voor Wetenschaplijk Onderzoek (NWO).

*Electronic address: K.Gubbels@phys.uu.nl

[1] D. M. Eagles, Phys. Rev. 186, 456 (1969).

[2] A.J. Leggett, in Modern Trends in the Theory of Condensed Matter (Springer-Verlag, Berlin, 1980), p. 13.

[3] P. Nozières and S. Schmitt-Rink, J. Low Temp. Phys. 59, 195 (1985).

[4] M. W. Zwierlein et al., Science 311, 492 (2006).

[5] M. W. Zwierlein et al., Nature (London) 442, 54 (2006).

[6] G. B. Partridge et al., Science 311, 503 (2006).

[7] D. E. Sheehy and L. Radzihovsky, Phys. Rev. Lett. 96, 060401 (2006).

[8] J. Kinnunen et al., Phys. Rev. Lett. 96, 110403 (2006).

[9] F. Chevy, Phys. Rev. Lett. 96, 130401 (2006); cond-mat/ 0605751.

[10] P. Pieri and G. C. Strinati, Phys. Rev. Lett. 96, 150404 (2006).

[11] W. Yi and L.-M. Duan, Phys. Rev. A 73, 031604(R) (2006).

[12] T. N. De Silva and E. J. Mueller, Phys. Rev. A 73, 051602(R) (2006); Phys. Rev. Lett. 97, 070402 (2006).

[13] M. Haque and H. T. C. Stoof, Phys. Rev. A 74, 011602(R) (2006).

[14] T.-L. Ho and H. Zhai, cond-mat/0602568.

[15] A. Imambekov et al., cond-mat/0604423 [Phys. Rev. A (to be published)].

[16] K. Machida et al., Phys. Rev. Lett. 97, 120407 (2006).

[17] L. M. Jensen et al., cond-mat/0604424.

[18] J.-P. Martikainen, Phys. Rev. A 74, 013602 (2006).

[19] C.-C. Chien et al., Phys. Rev. Lett. 97, 090402 (2006); Phys. Rev. A 74, 021602(R) (2006).

[20] M. M. Parish et al., cond-mat/0605744.

[21] A. Bulgac and M. McNeil Forbes, cond-mat/0606043.

[22] G. Sarma, J. Phys. Chem. Solids 24, 1029 (1963).

[23] R. Combescot and C. Mora, Europhys. Lett. 68, 79 (2004).

[24] After we submitted this Letter, Shin et al. [Phys. Rev. Lett. 97, 030401 (2006)] reported the observation of a shell structure in a trapped unbalanced Fermi gas that is consistent with the shell structure of the Sarma phase. However, Shin et al. interpret their finding as evidence for phase separation, although there is no indication of a first-order transition in the data presented.

[25] M. Houbiers et al., Phys. Rev. A 56, 4864 (1997).

[26] B. M. Fregoso and G. Baym, Phys. Rev. A 73, 043616 (2006).

[27] G.E. Astrakharchik et al., Phys. Rev. Lett. 93, 200404 (2004).

[28] J. Carlson and S. Reddy, Phys. Rev. Lett. 95, 060401 (2005). 